\title{
Relaxation dynamics in suspensions of ferromagnetic particles
}

\author{
M.C. Miguel and J.M. Rubí \\ Departament de Física Fonamental, Facultat de Física Universitat de Barcelona, Diagonal 647, 08028 Barcelona, Spain
}

(Received 22 June 1994; revised manuscript received 19 October 1994)

\begin{abstract}
We have studied the relaxation dynamics of a dilute assembly of ferromagnetic particles in suspension. A formalism based on the Smoluchowski equation, describing the evolution of the probability density for the directions of the magnetic moment and of the axis of easy magnetization of the particles, has been developed. We compute the rotational viscosity from a Green-Kubo formula and give an expresion for the relaxation time of the particles which comes from the dynamic equations of the correlation functions. Concerning the relaxation time for the particles, our results agree quite well with experiments performed on different samples of ferromagnetic particles for which the magnetic energy, associated with the interaction between the magnetic moments and the external field, or the energy of anisotropy plays a dominant role.
\end{abstract}

PACS number(s): $62.10 .+\mathrm{s}, 83.80 . \mathrm{Gv}, 75.50 . \mathrm{Mm}, 05.40 .+\mathrm{j}$

\section{INTRODUCTION}

Systems of single-domain ferromagnetic particles immersed in a solid or liquid phase exhibit a number of interesting relaxation phenomena which have been the subject matter of many experimental and theoretical analyses $[1,2]$. These phenomena are essential in the study of the dynamics of these particles, and have a clear influence when determining the transport coefficients; in particular, the effective viscosity. One of the main peculiarities of these systems is that their properties are greatly influenced by the presence of an external magnetic field. It is precisely this fact which has been the basis of many practical applications [3].

The rotational dynamics of a ferromagnetic particle embedded in a solid or liquid phase is the result of the competition of three orientational mechanisms related to the external field, the axis of easy magnetization, and Brownian motion, which acts only when the particle is suspended in a fluid phase. That is, whereas the magnetic moment of the ferromagnetic particle relaxes towards the direction of the magnetic field, the axis of easy magnetization tends to be aligned with the magnetic moment, thus giving rise to different coupled relaxation phenomena. Until recently, the most frequent case that has been studied in the literature deals with rigid dipoles [4], for which the anisotropy energy is dominant due to the large value of the anisotropy constant, and because the radius of the particle usually exceeds a critical value. Under these conditions, the relaxation of the axis of easy magnetization towards the magnetic moment is inhibited and both vectors relax together. However, there are materials for which the anisotropy energy may be comparable to the energy associated with the interaction with the magnetic field, or even smaller. Therefore a general theory encompassing such a wide variety of situations and accounting for experimental results should be developed. The presence of different relaxation mechanisms has implications in the form of the effective viscosity of the system, which exhibits significant corrections when compared to the viscosity of a suspension of nonmagnetic particles of the same shape. Another point of interest is the appearance of relaxation times which can be measured by means of birefringence experiments.

The purpose of this paper is to present a theory capable of giving expressions for the relevant transport coefficients of the system and of the characteristic relaxation times. We will focus on the general situation in which the magnetic and anisotropy energies of the particles may have arbitrary values. The formalism we have developed is based on the linear response theory where the correlation dynamics comes from a Smoluchowski equation. As we will show throughout the paper, our results for the relaxation time of the rotation of the particle agree quite well with birefringence experiments.

We have distributed the paper in the following way: in Sec. II, we establish basic equations describing the dynamics of the degrees of freedom. Of particular interest is the Smoluchowski equation for the probability density which is given in a general case (for unspecified values of the magnetic and anisotropy energies), and can be compared to another one previously obtained from a different theoretical method. In Sec. III we deal with the calculation of the rotational viscosity using a GreenKubo equation proposed from the linear response theory. This method leads to an expression for this transport coefficient which is studied in particular situations of interest. Section IV is devoted to the calculation of the relaxation time of the particles, considering the different orientational mechanisms. We have compared our results to experiments done when the magnetic energy or the energy of anisotropy are dominant, obtaining a good agreement in both situations. Finally, in the last section we summarize our main results.

\section{COUPLED DYNAMICS OF THE DEGREES OF FREEDOM}

We consider a dilute suspension of spherical singledomain ferromagnetic particles under the action of an 
external magnetic field. The energy of each particle is the sum of two contributions. These contributions originate from the externally imposed magnetic field and the presence of an axis of easy magnetization (for uniaxial crystals). Its expression is given by

$$
U=-\vec{m} \cdot \vec{H}-K_{a} V_{p}(\hat{n} \cdot \hat{R})^{2},
$$

where $\vec{m}=m_{0} \hat{R}$ is the magnetic moment of the particles, with $m_{0}$ the magnetic moment strength, $\vec{H}$ is the external magnetic field, $K_{a}$ is the first anisotropy constant (assumed positive), $V_{p}$ is the volume of one of these spheres, and $\hat{n}$ is the unit vector along the direction of the axis of easy magnetization for uniaxial magnetic crystals. It is clear from Eq. (2.1) that in the general case where both contributions may take arbitrary values, the relaxation mechanisms of the degrees of freedom, $\hat{R}$ and $\hat{n}$, of the ferromagnetic spheres in suspension are coupled.

The deterministic dynamics of $\hat{R}$ is governed by the Landau-Gilbert equation [5], proposed to study the relaxation of the magnetic moments of magnetic particles embedded in a solid matrix

$$
\frac{d \hat{R}}{d t}=-\frac{\gamma_{0}}{m_{0}} \frac{\partial U}{\partial \hat{R}} \times \hat{R}-\frac{\alpha}{m_{0}} \frac{d \hat{R}}{d t} \times \hat{R} .
$$

From this equation, one may identify the two mechanisms responsible for the variation of $\hat{R}$ : the effective field $\vec{H}_{\text {eff }} \propto-\frac{\partial U}{\partial \hat{R}}$, which causes a Larmor precessional motion of $\hat{R}$, and the mean field, $\vec{H}_{d} \propto-\frac{d \hat{R}}{d t}$, which introduces a damping due to the collisions of the electrons determining the magnetic moment of the domain in a metal. In Eq. (2.2), $\gamma_{0}$ is the gyromagnetic ratio for an electron, and the quantity $\alpha$ plays the role of a damping coefficient.

The Landau-Gilbert equation can be rewritten such that

$$
\frac{d \hat{R}}{d t}=-h \hat{R} \times \frac{\partial U}{\partial \hat{R}} \times \hat{R}+\vec{\omega}_{L} \times \hat{R},
$$

with $\vec{\omega}_{L}=m_{0} g \vec{H}_{e f f}$ as the Larmor frequency of the precessional motion, and where $g \equiv \frac{\gamma_{0}}{m_{0}}\left[1+\left(\frac{\alpha}{m_{0}}\right)^{2}\right]^{-1}$ and $h \equiv \frac{\alpha \gamma_{0}}{m_{0}^{2}}\left[1+\left(\frac{\alpha}{m_{0}}\right)^{2}\right]^{-1}[1]$. This equation is valid in a stationary frame of reference. If the ferromagnetic particle is rotating itself with the angular velocity $\vec{\Omega}$, we must modify Eq. (2.3) by adding the corresponding contribution coming from the rotation on its right hand side. One then has

$$
\frac{d \hat{R}}{d t}=-h \hat{R} \times \frac{\partial U}{\partial \hat{R}} \times \hat{R}+\left(\vec{\omega}_{L}+\vec{\Omega}\right) \times \hat{R} .
$$

Furthermore, the dynamics of $\hat{n}$ follows from the kinematic relation

$$
\frac{d \hat{n}}{d t}=\vec{\Omega} \times \hat{n} .
$$

This expression can be rewritten as

$$
\frac{d \hat{n}}{d t}=\left(\vec{\omega}_{0}+\frac{1}{\xi_{r}} \vec{m} \times \vec{H}\right) \times \hat{n},
$$

with $\xi_{r}=8 \pi \eta_{0} a^{3}$ being the rotational friction coefficient of the particles; $\eta_{0}$ is the viscosity of the carrier fluid, $a$ is the hydrodynamic radius of the particles, and $\vec{\omega}_{0}$ the vorticity of the carrier fluid. One arrives at this expression after using the deterministic part of the balance equation for the total angular momentum

$$
I \frac{d \vec{\Omega}}{d t}+\gamma_{0}^{-1} \frac{d \vec{m}}{d t}=-\xi_{r}\left(\vec{\Omega}-\vec{\omega}_{0}\right)+\vec{m} \times \vec{H}+\vec{T}^{B},
$$

provided that we neglect the term accounting for the inertial effects and the term coming from the angular momentum of the electrons determining the magnetic moment of the particles. Here $\vec{T}^{B}$ is the Brownian torque acting on the particle [6].

The evolution of the probability density, $\psi(\underline{\gamma}, t)$, with $\underline{\gamma} \equiv(\hat{R}, \hat{n})$, is governed by the Smoluchowski equation. When $\vec{\Omega}=\overrightarrow{0}$, which corresponds to the case of particles embedded in a solid matrix, the Smoluchowski equation was deduced by Brown [7] from the Landau-Gilbert equation introducing a stochastic Langevin source. Shliomis and co-workers [8] obtained this equation for a suspension of rigid dipoles. If we define the dimensionless parameters $\mu=\frac{m_{0} H}{k_{B} T}$ and $\sigma=\frac{K_{a} V_{p}}{k_{B} T}$, comparing magnetic and anisotropy energies to thermal energy, respectively, this last situation corresponds to the limit $\sigma \gg \mu$. Raikher and Shliomis [9] also proposed the Smoluchowski equation for the opposite limit $\sigma \ll \mu$, in which the dipoles are rapidly oriented towards the field direction. As regards the general case for arbitrary values of the ratio $\mu / \sigma$, Shliomis and co-workers also deduced the appropriate Smoluchowski equation from a model similar to the itinerant oscillator model [10-12]. In such a general situation, the Smoluchowski equation is given by

$$
\frac{\partial \psi}{\partial t}=\frac{\partial}{\partial \underline{\gamma}} \cdot\left(\underline{D} \cdot \frac{\partial}{\partial \underline{\gamma}} \psi-\psi \underline{b} \cdot \frac{\partial U}{\partial \underline{\gamma}}\right),
$$

where $\underline{D}$ and $\underline{b}$ are the diffusion and the mobility matrices, respectively, related through the Einstein relation, $\underline{D}=k_{B} T \underline{b}$. Taking into account Eqs. (2.4) and (2.6), we can obtain the expressions for the mobility matrix and after some mathematical transformations, we can rewrite the Smoluchowski equation as

$$
\begin{aligned}
\frac{\partial \psi}{\partial t}= & D_{r}\left(\overrightarrow{\mathcal{R}}_{\hat{\boldsymbol{R}}}+\overrightarrow{\mathcal{R}}_{\hat{n}}\right) \cdot\left(\psi\left(\overrightarrow{\mathcal{R}}_{\hat{R}}+\overrightarrow{\mathcal{R}}_{\hat{n}}\right) \frac{U}{k_{B} T}\right. \\
& \left.+\left(\overrightarrow{\mathcal{R}}_{\hat{R}}+\overrightarrow{\mathcal{R}}_{\hat{n}}\right) \psi\right)+D_{m} \overrightarrow{\mathcal{R}}_{\hat{R}} \cdot\left(\psi \overrightarrow{\mathcal{R}}_{\hat{R}} \frac{U}{k_{B} T}+\overrightarrow{\mathcal{R}}_{\hat{R}} \psi\right) \\
& -\overrightarrow{\mathcal{R}}_{\hat{\boldsymbol{R}}} \cdot\left(\vec{\omega}_{L} \psi\right)-\left(\overrightarrow{\mathcal{R}}_{\hat{\boldsymbol{R}}}+\overrightarrow{\mathcal{R}}_{\hat{n}}\right) \cdot\left(\vec{\omega}_{0} \psi\right)
\end{aligned}
$$

where $\overrightarrow{\mathcal{R}}_{\hat{n}} \equiv \hat{n} \times \frac{\partial}{\partial \hat{n}}$ and $\overrightarrow{\mathcal{R}}_{\hat{R}} \equiv \hat{R} \times \frac{\partial}{\partial \hat{R}}$ are rotational operators, $D_{r} \equiv \frac{k_{B} T}{\xi_{r}}$ is the Brownian rotational diffusion coefficient, and $\stackrel{\text { s }}{D}_{m} \equiv k_{B} T h$ can be interpreted as the diffusion coefficient of the magnetic moment inside the particles. These diffusion coefficients are related to two relaxation times involved in the Smoluchowski equation, namely $\tau_{0}=\left(2 D_{m}\right)^{-1}$ related to the decay of $\vec{m}$ towards 
the effective field, and the Brownian time $\tau_{B}=\left(2 D_{r}\right)^{-1}$. The Smoluchowski equation (2.9) agrees with the corresponding one obtained in Ref. [10] by using a model similar to the itinerant oscillator model and will be used in our subsequent analysis.

\section{GREEN-KUBO FORMULA FOR THE ROTATIONAL VISCOSITY}

In this section, we will focus on the determination of the rotational viscosity from the corresponding GreenKubo formula. This formula gives this transport coefficient in terms of the correlation function of the axial vector, $\vec{\Pi}_{p}^{(a)}$, related to the antisymmetric part of the contribution of the particles to the pressure tensor [6],

$$
\eta_{r}=\frac{1}{V k_{B} T} \int_{0}^{\infty} d t\left\langle\Pi_{p, y}^{(a)}(t) \Pi_{p, y}^{(a)}(0)\right\rangle,
$$

where $V$ is the volume of the system.

In Ref. [6], we obtained a relationship between the particle contribution to the pressure tensor, $\stackrel{\leftrightarrow}{\mathbf{\Pi}}_{p}$, and the external torque experienced by the particle during its motion. As a result, if we consider the magnetic field pointing towards the $z$ direction, it is found that the rotational viscosity can be finally rewritten as

$$
\eta_{r}=\frac{3}{2} \phi \eta_{0} D_{r} \mu^{2} \int_{0}^{\infty} d t\left\langle R_{x}(t) R_{x}(0)\right\rangle,
$$

with $\phi=\frac{4 / 3 \pi a^{3}}{V}$ being the volume fraction of particles.

To proceed further, we need to know the expression for the correlation function appearing in Eq. (3.2). This correlation function follows from the general prescription

$$
\left\langle R_{x}(t) R_{x}(0)\right\rangle=\int d \hat{R} d \hat{R}^{\prime} d \hat{n} d \hat{n}^{\prime} R_{x} R_{x}^{\prime} \psi\left(\hat{R}, \hat{n}, t ; \hat{R}^{\prime}, \hat{n}^{\prime}, 0\right),
$$

with $\psi\left(\hat{R}, \hat{n}, t ; \hat{R}^{\prime}, \hat{n}^{\prime}, 0\right)$ being the joint probability, which, in the most general case, is a function of both the magnetic moment orientation $\hat{R}$ and the particle orientation $\hat{n}$. For a Markov process, this probability satisfies the same Smoluchowski equation as the one governing the evolution of the conditional probability. From the Smoluchowski equation (2.9) we then obtain the evolution equation for the correlation $\left\langle R_{x}(t) R_{x}(0)\right\rangle$,

$$
\begin{aligned}
\frac{1}{D_{r}} \frac{d}{d t}\left\langle R_{x}(t) R_{x}(0)\right\rangle= & \left(1+\frac{D_{m}}{D_{r}}\right)\left\{-2\left\langle R_{x}(t) R_{x}(0)\right\rangle\right. \\
& \left.-\mu\left\langle\left(R_{x} R_{z}\right)(t) R_{x}(0)\right\rangle\right\} \\
& +\frac{D_{m}}{D_{r}} 2 \sigma\left\{\left\langle n_{x}(\hat{n} \cdot \hat{R})\right](t) R_{x}(0)\right\rangle \\
& \left.-\left\langle\left[R_{x}(\hat{n} \cdot \hat{R})^{2}\right](t) R_{x}(0)\right\rangle\right\},
\end{aligned}
$$

where for the sake of simplicity, we have used the shorthand notation $(\cdots)(t)$ to indicate that each component of the unitary vectors $\hat{R}$ and $\hat{n}$ inside the brackets is a function of time. At this point, it is interesting to realize that the right hand side of Eq. (3.4) involves other unknown correlation functions, whose evolution equations also come from the Smoluchowski equation (2.9). Instead of a closed equation, we then obtain a hierarchy of differential equations for the correlation functions. To arrive at an explicit solution, we must introduce decoupling approximations, as is usually done when dealing with the dynamics of complex systems [14] or with stochastic processes [12]. In our problem, the underlying idea to carry out the different decouplings comes from the approximation of statistical independency of the fluctuation dynamics of the quantities which vanish at equilibrium, when averaged, and those which are different from zero. Another aspect that should be kept in mind when performing such decouplings is the invariance under reflections of the easy axis of magnetization, $\hat{n}$, from which we cannot separate $\langle\hat{n} \cdots \hat{n}\rangle$ into odd powers of $\hat{n}$. As an example, the correlation $\left\langle\left(R_{x} R_{z}\right)(t) R_{x}(0)\right\rangle$ may be approximated by

$$
\left\langle\left(R_{x} R_{z}\right)(t) R_{x}(0)\right\rangle \sim\left\langle R_{x}(t) R_{x}(0)\right\rangle\left\langle R_{z}\right\rangle_{e q} .
$$

This approximation may be justified from the fact that in equilibrium both quantities are not correlated, $\left\langle\left(R_{x} R_{z}\right)\right\rangle_{e q}=0$; moreover, $\left\langle R_{z}\right\rangle_{e q} \neq 0$, whereas $\left\langle R_{x}\right\rangle_{e q}=$ 0 . After perturbing the system we will assume that these quantities remain uncorrelated. Furthermore, linearization in time has been taken into account and consequently $\left\langle R_{z}(t)\right\rangle \sim\left\langle R_{z}\right\rangle_{e q}$ is approximated to its equilibrium value for small perturbations. In the Appendix, we indicate all of these decouplings, as well as the appropriate linearizations.

The quantity $\left\langle\left[n_{x}(\hat{n} \cdot \hat{R})\right](t) R_{x}(0)\right\rangle$, appearing in Eq. (3.4), cannot be directly decoupled and, therefore, must be studied separately. Its corresponding evolution equation can also be obtained from the Smoluchowski equation (2.9). One has

$$
\begin{aligned}
\frac{1}{D_{r}} \frac{d}{d t}\left\langle\left[n_{x}(\hat{n} \cdot \hat{R})\right](t) R_{x}(0)\right\rangle= & -\mu\left\langle\left[R_{x}\left(n_{z}(\hat{n} \cdot \hat{R})\right)\right](t) R_{x}(0)\right\rangle-2\left\langle\left[n_{x}(\hat{n} \cdot \hat{R})\right](t) R_{x}(0)\right\rangle \\
& +\frac{D_{m}}{D_{r}}\left(\mu\left\langle\left(n_{x} n_{z}\right)(t) R_{x}(0)\right\rangle-\mu\left\langle\left[n_{x}\left(R_{z}(\hat{n} \cdot \hat{R})\right)\right](t) R_{x}(0)\right\rangle\right. \\
& \left.-2\left\langle\left[n_{x}(\hat{n} \cdot \hat{R})\right](t) R_{x}(0)\right\rangle+2 \sigma\left\langle\left\{n_{x}(\hat{n} \cdot \hat{R})\left[1-(\hat{n} \cdot \hat{R})^{2}\right]\right\}(t) R_{x}(0)\right\rangle\right) .
\end{aligned}
$$

Following the same line of reasoning, we decouple some of the quantities appearing in this equation. However, the correlation $\left\langle\left(n_{x} n_{z}\right)(t) R_{x}(0)\right\rangle$ cannot be approximated in the same way as we have done with the other quantities. Thus we must also consider its dynamic equation

$$
\begin{aligned}
\frac{1}{D_{r}} \frac{d}{d t}\left\langle\left(n_{x} n_{z}\right)(t) R_{x}(0)\right\rangle= & \mu\left\langle\left[n_{x}(\hat{n} \cdot \hat{R})\right](t) R_{x}(0)\right\rangle-\mu\left\langle\left(n_{z}^{2} R_{x}\right)(t) R_{x}(0)\right\rangle \\
& -\mu\left\langle\left(n_{x} n_{z} R_{z}\right)(t) R_{x}(0)\right\rangle-6\left\langle\left(n_{x} n_{z}\right)(t) R_{x}(0)\right\rangle
\end{aligned}
$$


whose correlations can be decoupled as indicated in the Appendix.

The procedure that has been followed enables us to write a closed system of just three differential equations for the correlation functions $\left\langle R_{x}(t) R_{x}(0)\right\rangle,\left\langle\left[n_{x}(\hat{n}\right.\right.$. $\left.\hat{R})](t) R_{x}(0)\right\rangle$, and $\left\langle\left(n_{x} n_{z}\right)(t) R_{x}(0)\right\rangle$. These are the only independent quantities in our approximation to the problem. After Laplace transforming, we can write the set of differential equations (3.4), (3.6), and (3.7) in the following way:

$$
\underline{\mathcal{A}} \cdot \overrightarrow{\mathcal{R}}=D_{r}^{-1} \overrightarrow{\mathcal{R}}^{0},
$$

where the components of the vector $\overrightarrow{\mathcal{R}}=\left(\mathcal{R}_{1}, \mathcal{R}_{2}, \mathcal{R}_{3}\right)$ are the three Laplace transforms of $\left\langle R_{x}(t) R_{x}(0)\right\rangle,\left\langle\left[n_{x}(\hat{n}\right.\right.$. $\left.\hat{R})](t) R_{x}(0)\right\rangle$, and $\left\langle\left(n_{x} n_{z}\right)(t) R_{x}(0)\right\rangle$, respectively. $\overrightarrow{\mathcal{R}}^{0}=$ $\left(\mathcal{R}_{1}^{0}, \mathcal{R}_{2}^{0}, \mathcal{R}_{3}^{0}\right)$ is a vector whose components are the initial values of these correlation functions

$$
\mathcal{R}_{1}^{0} \equiv\left\langle R_{x}^{2}(0)\right\rangle=\frac{\mathcal{L}(\mu)}{\mu},
$$

$$
\begin{gathered}
\mathcal{R}_{2}^{0} \equiv\left\langle\left[n_{x} R_{x}(\hat{n} \cdot \hat{R})\right](0)\right\rangle=\frac{\mathcal{L}(\mu)}{\mu} Q(\sigma), \\
\mathcal{R}_{3}^{0} \equiv\left\langle\left(n_{x} R_{x} n_{z}\right)(0)\right\rangle=\frac{1}{2 \mu}\left(1-3 \frac{\mathcal{L}(\mu)}{\mu}\right)(3 Q(\sigma)-1),
\end{gathered}
$$

where $\mathcal{L}(\mu)=\operatorname{coth} \mu-\frac{1}{\mu}$ is the Langevin function and $Q(\sigma)=\frac{1}{2 \sigma}\left(\frac{2 e^{\sigma}}{\sqrt{\frac{\pi}{\sigma}} \operatorname{Erfi(\sqrt {\sigma })}}-1\right)$. These initial values are determined after averaging with the equilibrium distribution function

$$
\Psi_{e q}=\frac{1}{8 \pi^{5 / 2} \frac{\sinh \mu}{\mu} \frac{\operatorname{Erfi(\sqrt {\sigma })}}{\sqrt{\sigma}}} \exp \left[\mu(\hat{R} \cdot \hat{H})+\sigma(\hat{n} \cdot \hat{R})^{2}\right] .
$$

The matrix $\underline{\mathcal{A}} \equiv\left(A_{i j}\right)$ is found to be

$\left(A_{i j}\right)=\left(\begin{array}{ccc}\frac{s}{D_{r}}+2+\mu \mathcal{L}(\mu)+\frac{D_{m}}{D_{r}}[2+\mu \mathcal{L}(\mu)+2 \sigma Q(\sigma)] & -2 \frac{D_{m}}{D_{r}} \sigma & 0 \\ \mu \mathcal{L}(\mu) Q(\sigma) & \frac{s}{D_{r}}+2+\frac{D_{m}}{D_{r}}\left\{[2+\mu \mathcal{L}(\mu)]+\left(\frac{1}{Q(\sigma)}-3\right)\right\} & -\frac{D_{m}}{D_{r}} \mu \\ \mathcal{L}(\mu)[1-3 Q(\sigma)]+\mu Q(\sigma) & -\mu & \frac{s}{D_{r}}+6+\mu \mathcal{L}(\mu)\end{array}\right)$

whose diagonal elements characterize the decay of each correlation function in the absence of coupled dynamics, and whose nondiagonal elements describe the coupling between the relaxation dynamics of such correlations.

The Laplace transform $\mathcal{R}_{1}(s)$ of the correlation $\left\langle R_{x}(t) R_{x}(0)\right\rangle$ is defined as

$$
\mathcal{R}_{1}(s)=\int_{0}^{\infty} d t e^{-s t}\left\langle R_{x}(t) R_{x}(0)\right\rangle
$$

From Eq. (3.2), we then conclude that

$$
\eta_{r}=\frac{3}{2} \phi \eta_{0} D_{r} \mu^{2} \lim _{s \rightarrow 0} \mathcal{R}_{1}(s)
$$

Consequently, after using Cramers's rule in Eq. (3.8) the rotational viscosity of the suspension is given by

$$
\frac{\eta_{r}}{\eta_{s a t}}=\mu^{2} \lim _{s \rightarrow 0} \frac{\left(A_{22} A_{33}-A_{23} A_{32}\right) \mathcal{R}_{1}^{0}-A_{12} A_{33} \mathcal{R}_{2}^{0}+A_{12} A_{23} \mathcal{R}_{3}^{0}}{\left|\left(A_{i j}\right)\right|}
$$

with $\eta_{s a t}=3 / 2 \eta_{0} \phi$ as the saturation value of the rotational viscosity and $\left|\left(A_{i j}\right)\right|$ the determinant of the matrix.

We have obtained an analytic expression for the rotational viscosity of a magnetic fluid in the general case where $\mu / \sigma$ may take arbitrary values and the orientation of the vectors $\hat{R}$ and $\hat{n}$ is random. The different terms in Eq. (3.16) are the matrix elements $\left(A_{i j}\right)$ characterizing the coupled relaxation dynamics of the three independent correlation functions $\left\langle R_{x}(t) R_{x}(0)\right\rangle,\left\langle\left[n_{x}(\hat{n} \cdot \hat{R})\right](t) R_{x}(0)\right\rangle$, and $\left\langle\left(n_{x} n_{z}\right)(t) R_{x}(0)\right\rangle$. Thus the rotational viscosity depends on these three correlation functions due to their coupled dynamics and, as we expected, it is not only a function of the parameters $\mu$ and $\sigma$, but also of the ratio $D_{m} / D_{r}$. This last dependence comes from the fact that any departure of the magnetic moment of the parti- cle from the equilibrium orientation is accompanied by a precession of the vector $\hat{R}$ with the corresponding dissipation of energy, as well as the dissipation due to the rotation of the particle in the viscous fluid. Previously, we have indicated that the parameter $D_{m}=k_{B} T h$ could be interpreted as a diffusion coefficient of the magnetic moment inside each particle. Thus, for a given value of the Brownian rotational diffusion coefficient $D_{r}$, the smaller the value of $D_{m}$ the greater is the dissipation of energy and consequently the rotational viscosity increases. This is exactly what we observe in Figs. 1 and 2.

Moreover, from Eq. (3.16) we can rederive the rotational viscosity of a magnetic fluid consisting of what is commonly named rigid dipoles $[4,6,8,13]$, for which $\mu \ll \sigma$ and $D_{m}=0$. It turns out to be 


$$
\frac{\eta_{r}}{\eta_{s a t}}=\frac{\mu-\tanh \mu}{\mu+\tanh \mu}
$$

In Ref. [6], we obtained this same result after performing just one decoupling, following the same criteria, in a much simpler equation. But, exactly the same result was derived phenomenologically by Shliomis [13]. He compared this result to an experiment measuring the viscosity of the magnetic fluid [15]. The result reproduced these experimental data, and consequently this formula has been extensively used in the literature. Later on, Martsenyuk et al. [8] tried to solve this simple case using the Smoluchowski equation formalism. They closed the infinite system of coupled equations with an effective-field approximation, and, although the result they obtained was close to Eq. (3.17), the phenomenological approach reproduced the experiments better. Based on this good result, we have solved this general situation with two degrees of freedom whose dynamics is coupled, following the same type of arguments. To our knowledge, there are no other theories providing an analytic expression for the viscosity, covering the whole range of values of the parameters $\mu$ and $\sigma$.

In Fig. 1, we represent the rotational viscosity versus the parameter $\mu$ for the rigid-dipole limit, for different values of the parameters $\sigma$ and $D_{m} / D_{r}$. At this point, it is interesting to discuss some limiting cases of Eq. (3.16) which have also been considered in the literature, specifically in Ref. [10]. For $\mu \ll 1$, Eq. (3.16) reduces to

$$
\frac{\eta_{r}}{\eta_{s a t}}=\frac{\mu^{2}}{6} F(\sigma)
$$

where the function $F(\sigma)$ has been defined as

$$
F(\sigma) \equiv \frac{1+\frac{D_{m}}{2 D_{r}}\left[\left(\frac{1}{Q(\sigma)}-1\right)+2 \sigma Q(\sigma)\right]}{\left\{1+\frac{D_{m}}{D_{r}}[1+\sigma Q(\sigma)]\right\}\left[1+\frac{D_{m}}{2 D_{r}}\left(\frac{1}{Q(\sigma)}-1\right)\right]}
$$

From expression (3.18), we conclude that for any value of the parameter $\sigma$, the rotational viscosity tends to zero as a second order power of the magnetic field strength. In particular, we can restrict ourselves to the cases where $\sigma \rightarrow 0$ and $\sigma \rightarrow \infty$. In these limits, we obtain

$$
\frac{\eta_{r}}{\eta_{s a t}}=\frac{\mu^{2}}{6} \frac{1}{\left(1+\frac{D_{m}}{D_{r}}\right)}
$$

and

$$
\frac{\eta_{r}}{\eta_{s a t}}=\frac{\mu^{2}}{6}
$$

respectively. These values agree quite well with the corresponding expressions given in Ref. [10], and with the results collected in Refs. [6,8], for the rigid-dipole conditions $(\sigma \rightarrow \infty)$.

In addition, from Fig. 1, we observe that the initial slope of these curves depends on the ratio $D_{m} / D_{r}$, in such a way that the greater the ratio $D_{m} / D_{r}$ the greater the value of $\mu$ until reaching the saturation limit corresponding to a given value of $\sigma$.

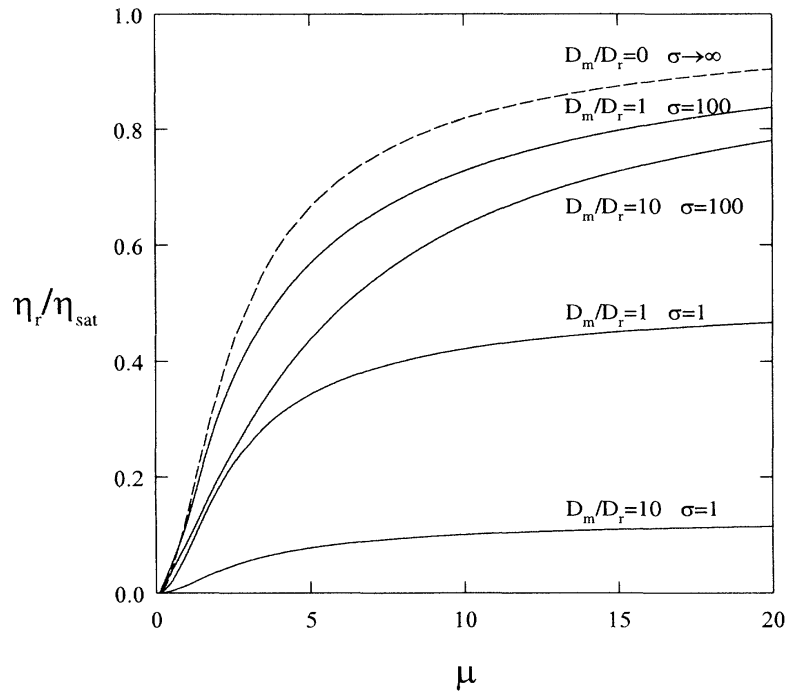

FIG. 1. Reduced rotational viscosity versus the parameter $\mu$ for different values of the ratio $D_{m} / D_{r}$ and of the anisotropy parameter, as indicated in the plot. The dashed line corresponds to the rigid-dipole limit.

Regarding the limit $\mu \gg 1$, Eq. (3.16) can also be studied when $\sigma \rightarrow 0$ and $\sigma \rightarrow \infty$. We obtain

$$
\frac{\eta_{r}}{\eta_{s a t}}=\frac{1}{\left(1+\frac{D_{m}}{D_{r}}\right)}
$$

and

$$
\frac{\eta_{r}}{\eta_{s a t}}=1
$$

respectively, which also coincide with the results given in Ref. [10]. Even in this case, with $\mu \gg 1$, if $\sigma$ takes moderate or small values, the rotational viscosity does not reach its saturation value any longer. In particular, if $\sigma \rightarrow 0$,

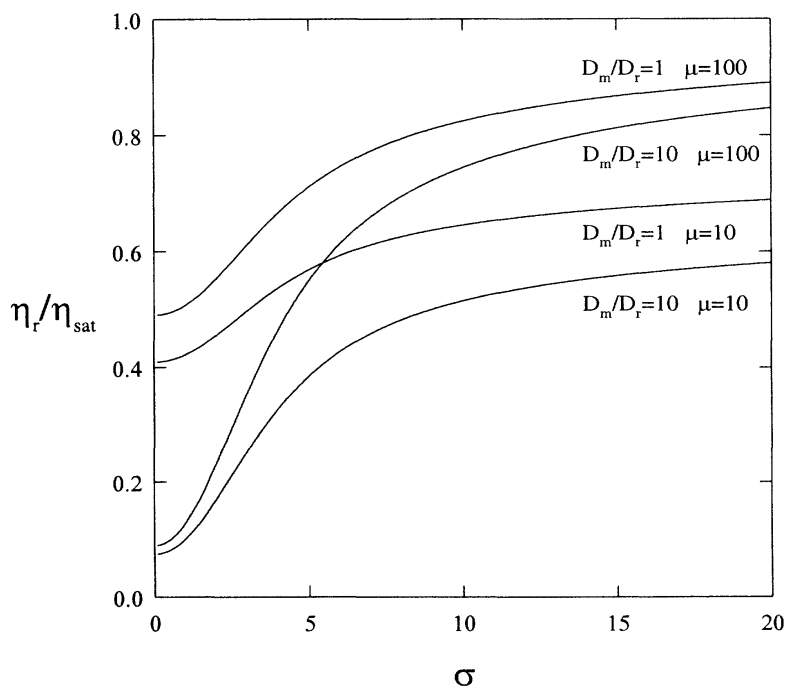

FIG. 2. Rotational viscosity versus the parameter $\sigma$ for different values of the ratio $D_{m} / D_{r}$ and of the parameter $\mu$. 
this saturation value depends on the ratio $D_{m} / D_{r}$ as we have pointed out in Eq. (3.22). That is, the dissipation not only depends on the solvent viscosity, but also on the damping constant $\alpha$ and the gyromagnetic factor $\gamma_{0}$. Additionally, the rotational viscosity increases with $\sigma$ until it reaches its saturation value when $\sigma \rightarrow \infty$.

The behavior of $\eta_{r} / \eta_{s}$ as a function of $\sigma$, and for different values of $\mu$, is depicted in Fig. 2, from which we can corroborate the main features of our previous analysis. The ratio $D_{m} / D_{r}$ has been obtained after considering the following values of the involved quantities: $\alpha / m_{0} \sim 10^{-2}$, $\gamma_{0} \sim 10^{7} \mathrm{G}^{-1} \mathrm{~s}^{-1}, M_{s} \sim 10^{3} \mathrm{G}, \eta_{0} \sim 10^{-2} \mathrm{~g} / \mathrm{cm} \mathrm{s}$, and the fact that the magnetic volume is almost the same as the hydrodynamic volume of the particles, with $a=10^{-6}$ cm.

An alternative procedure used to calculate the viscosity involves a rheological equation of state for the pressure tensor. In this way, it is also possible to obtain exactly the same expression for the rotational viscosity (3.16), but now in the presence of a linear vorticity field in stationary conditions.

\section{RELAXATION TIMES. COMPARISON WITH EXPERIMENTS}

The relaxation time associated with the orientation mechanism of the particles, $\tau_{R}$, has been measured recently by Bacri et al. $[16,17]$. This quantity comes from the relaxation time of the light intensity collected in a photocell after crossing the sample in the presence of an external magnetic field, and when applying additional pulses of magnetic field to perturb the sample. These experiments permit us to distinguish the different relaxation regimes occurring when the size and the nature of the magnetic material of the particles are modified. These regimes are determined by the parameters $\mu$ and $\sigma$, comparing magnetic and anisotropy energies with thermal energy, respectively.

In the experiments, it was observed that ferrofluid particles, prevented from moving by being quenched in a tight gel network, do not exhibit birefringence although they still show magnetization. Consequently, the birefringence of the solution is closely related to a mechanical alignment of the particles along the equilibrium orientation.

In Refs. $[6,13,18]$, two opposite limits were considered, one where $\sigma \gg \mu$ (rigid-dipole approximation) and another for which $\sigma \ll \mu$. For the former limit, the relaxation time for the perpendicular component of the magnetization is discovered to be $[6,13]$

$$
\tau=D_{r}^{-1}[2+\mu \mathcal{L}(\mu)]^{-1},
$$

which, in the case when $\mu \rightarrow \infty$, tends to $\frac{1}{D_{r} \mu}=\frac{2 \tau_{B}}{\mu}$. In the latter case, relaxation occurs in two steps, first a quick relaxation of $\hat{R}$ towards $\hat{H}$, then a mechanical rotation of the particle to the equilibrium orientation with the easy axis of magnetization parallel to $\hat{R}$ and $\hat{H}$. Under these conditions, the characteristic relaxation time could be obtained from the equation of motion of whatever component of the correlation function $\left\langle\left(\hat{n}_{\perp} n_{z}\right)(t)\left(\hat{n}_{\perp} n_{z}\right)(0)\right\rangle[18]$. Performing the corresponding decouplings in its evolution equation, we obtain the relaxation time

$$
\tau=\left(2 D_{r}\right)^{-1}\left(\sigma+\frac{1}{Q(\sigma)}\right)^{-1}=\tau_{B}\left(\sigma+\frac{1}{Q(\sigma)}\right)^{-1}
$$

which for $\sigma \rightarrow \infty$ tends to $\frac{1}{2 D_{r} \sigma}=\frac{\tau_{B}}{\sigma}$.

In the present analysis, we consider the less stringent case for which the ratio $\mu / \sigma$ may take arbitrary values. Consequently, both parameters $\mu$ and $\sigma$ are expected to determine the relaxation time associated with the rotational relaxation of the particles.

As for the limit $\sigma \ll \mu$ discussed previously, the appropriate quantity for describing this mechanical relaxational motion is again a component of the correlation function $\left\langle\left(\hat{n}_{\perp} n_{z}\right)(t)\left(\hat{n}_{\perp} n_{z}\right)(0)\right\rangle$. From the Smoluchowski equation, we obtain its dynamic equation

$$
\begin{aligned}
\frac{1}{D_{r}} \frac{d\left\langle\left(n_{x} n_{z}\right)(t)\left(n_{x} n_{z}\right)(0)\right\rangle}{d t}= & \mu\left\langle\left[n_{x}(\hat{n} \cdot \hat{R})\right](t)\left(n_{x} n_{z}\right)(0)\right\rangle-\mu\left\langle\left(n_{z}^{2} R_{x}\right)(t)\left(n_{x} n_{z}\right)(0)\right\rangle \\
& -\mu\left\langle\left(n_{x} n_{z} R_{z}\right)(t)\left(n_{x} n_{z}\right)(0)\right\rangle-6\left\langle\left(n_{x} n_{z}\right)(t)\left(n_{x} n_{z}\right)(0)\right\rangle
\end{aligned}
$$

Proceeding along the same lines as in the preceding section, we arrive at a closed set of three differential equations for the correlation functions $\left\langle R_{x}(t)\left(n_{x} n_{z}\right)(0)\right\rangle,\left\langle\left[n_{x}(\hat{n} \cdot \hat{R})\right](t)\left(n_{x} n_{z}\right)(0)\right\rangle$, and $\left\langle\left(n_{x} n_{z}\right)(t)\left(n_{x} n_{z}\right)(0)\right\rangle$. It is worthwhile pointing out that the quantities at time $t$ appearing in the three independent correlation functions are the same as in the preceding section. For the sake of simplicity, we will introduce the vector $\overrightarrow{\mathcal{Q}}=\left(\mathcal{Q}_{1}, \mathcal{Q}_{2}, \mathcal{Q}_{3}\right)$, whose components are the Laplace transforms of $\left\langle R_{x}(t)\left(n_{x} n_{z}\right)(0)\right\rangle,\left\langle\left[n_{x}(\hat{n} \cdot \hat{R})\right](t)\left(n_{x} n_{z}\right)(0)\right\rangle$, and $\left\langle\left(n_{x} n_{z}\right)(t)\left(n_{x} n_{z}\right)(0)\right\rangle$, respectively; and the vector $\overrightarrow{\mathcal{Q}}^{0}=\left(\mathcal{Q}_{1}^{0}, \mathcal{Q}_{2}^{0}, \mathcal{Q}_{3}^{0}\right)$ representing the initial values of these correlation functions. These initial values can be calculated with the equilibrium probability density at $t=0$. The system of differential equations can be written in matrix notation as

$$
\underline{\mathcal{A}} \cdot \overrightarrow{\mathcal{Q}}=D_{r}^{-1} \overrightarrow{\mathcal{Q}}^{0}
$$

with $\underline{\mathcal{A}}$ the coefficient matrix (3.13). We are particularly interested in the relaxation dynamics of $\left\langle\left(n_{x} n_{z}\right)(t)\left(n_{x} n_{z}\right)(0)\right\rangle$. From Eq. (4.4), we obtain its Laplace transform

$$
\mathcal{Q}_{3}(s)=D_{r}^{-1} \frac{\left(A_{21} A_{23}-A_{31} A_{22}\right) \mathcal{Q}_{1}^{0}+\left(A_{12} A_{31}-A_{11} A_{23}\right) \mathcal{Q}_{2}^{0}+\left(A_{11} A_{22}-A_{21} A_{12}\right) \mathcal{Q}_{3}^{0}}{\left|\left(A_{i j}\right)\right|}
$$


from which we can identify the relaxation time we are interested in,

$$
\tau_{R}=D_{r}^{-1} \lim _{s \rightarrow 0} \frac{\mathcal{Q}_{3}(s)}{\mathcal{Q}_{3}(0)} .
$$

In Fig. 3, we have represented the relaxation time $\tau_{R}$ versus $\mu$ for different ferromagnetic samples, but, in order to compare with experimental data from Ref. [16], we have also represented the relaxation time $\tau_{R}$ versus $H^{-1}$ in Fig. 4. The data correspond to two samples of magnetic particles of the same mean size but made of different magnetic materials, namely, $\mathrm{CoFe}_{2} \mathrm{O}_{4}$ and $\gamma-\mathrm{Fe}_{2} \mathrm{O}_{3}$. The Co-ferrite sample has an anisotropy constant $K=2 \times 10^{5} \mathrm{~J} / \mathrm{m}^{3}$, and the saturation value of the magnetization is $M_{s} \simeq 250 \mathrm{kA} / \mathrm{m}$. For the maghemite, the anisotropy constant is $K=4 \times 10^{3} \mathrm{~J} / \mathrm{m}^{3}$ and $M_{s} \simeq 270 \mathrm{kA} / \mathrm{m}$. With these values, the Co-ferrite sample can be considered as a rigid dipole $(\sigma \gg \mu)$. On the other hand, the maghemite particles are such that $\mu>\sigma$. Regarding the values of the anisotropy constant $\sigma$ and the ratio $m_{0} / k_{B} T=\mu_{0} M_{s} V_{m} / k_{B} T$, we have taken $\sigma \sim 15, m_{0} / k_{B} T \sim 2.8 \times 10^{-4} \mathrm{~m} / \mathrm{A}$ for the maghemite and $\sigma \sim 565, m_{0} / k_{B} T \sim 1.8 \times 10^{-4} \mathrm{~m} / \mathrm{A}$ for the Coferrite. For both samples, the values of the remaining quantities are $\tau_{B} \sim 4.5 \mathrm{~ms}$ and $D_{m} / D_{r} \sim 1$.

As it was observed in the experiments for the Co-ferrite sample, $\tau_{R}$ tends to zero when $H \rightarrow \infty$. Both $\hat{R}$ and $\hat{n}$ quickly relax towards the field direction due to the rigidity of the dipoles. For the maghemite sample $\tau_{R}$ tends to a fixed, nonvanishing value $(\sim 0.3)$, which can also be obtained from Eq. (4.2). Under these particular conditions, the magnetic moments rapidly relax towards the field direction, but due to the moderate value of $\sigma$, the relaxation of the easy axis of magnetization, $\hat{n}$, or in other words, the mechanical relaxation of the particles, takes place in a finite period of time.

Concerning the behavior of $\tau_{R}$ when $H \rightarrow 0$, we ob-

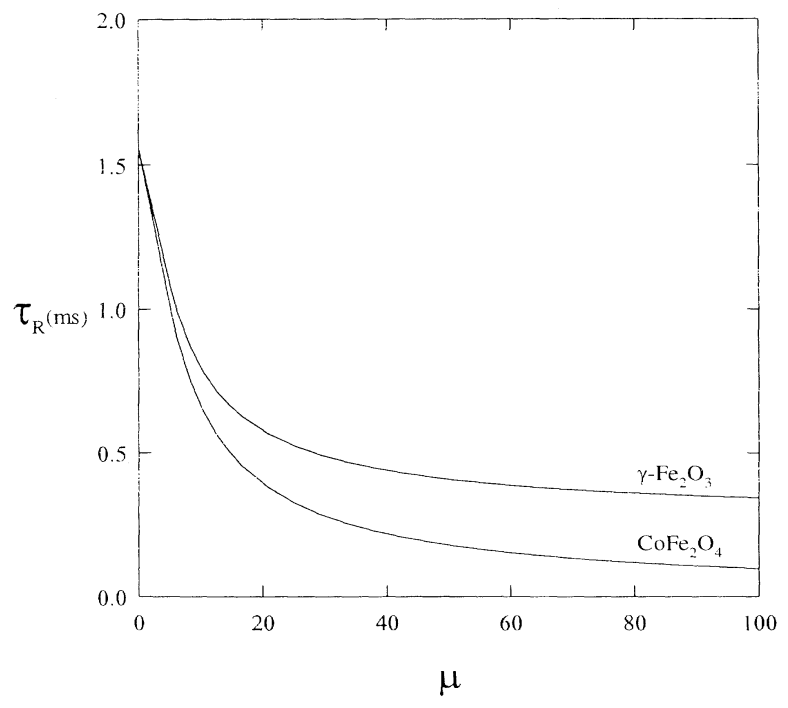

FIG. 3. Relaxation time of the particles as a function of $\mu$ for the Co-ferrite and maghemite samples.

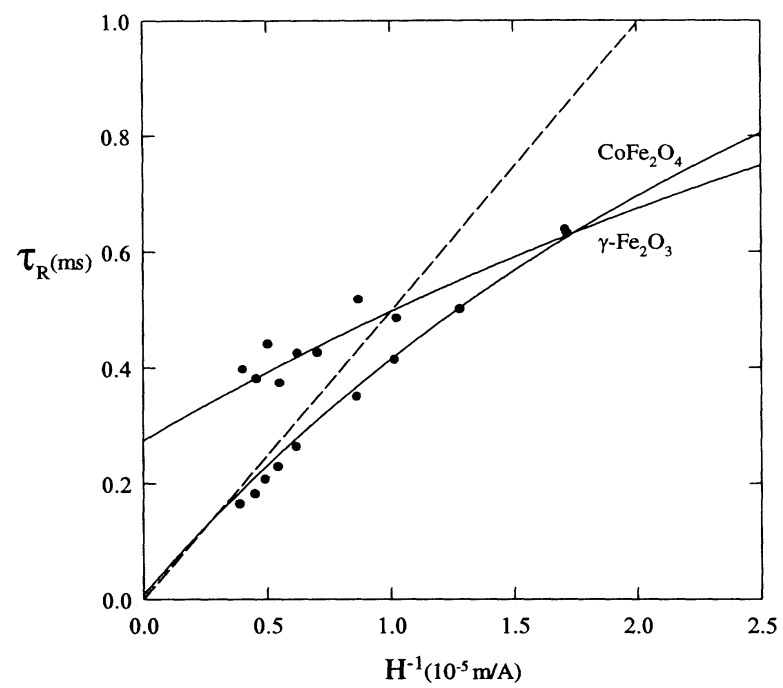

FIG. 4. Relaxation time of the particles as a function of $H^{-1}$ for the Co-ferrite and maghemite samples. Experimental data of Ref. [16] correspond to the dots. The dashed line corresponds to the limit $\mu \gg 1$ for the Co-ferrite, Ref. [19].

serve that $\tau_{R} \rightarrow \frac{1}{6 D_{r}}=\frac{\tau_{B}}{3}$ independently of the value of the parameter $\sigma$. This time corresponds to the wellknown characteristic relaxation time of the correlations of the components of the second order tensor $\left(\hat{n} \hat{n}-\frac{I}{3}\right)$ for a purely diffusive process [14].

In Fig. 4 we have also represented the extrapolation of the relaxation time coming from rather simple considerations made in Ref. [19] for the rigid-dipole limit under the action of a very large external magnetic field. Our results agree with the asymptotic behavior in its validity range, but at the same time, they show the deviations at intermediate and low magnetic field. These simple arguments can also be proposed for the opposite case $\mu \gg \sigma$ reproducing the asymptotic value $\tau_{R} \sim 0.3$ for the maghemite sample, but they are not able to explain the $\mu$ dependence of the relaxation time for this material.

\section{CONCLUSIONS}

In this paper, we have presented a general formalism to study the relaxation dynamics of ferromagnetic particles with the main purpose of providing explicit expressions for the viscosity and relaxation times which can be applied to different situations ranging from the rigid-dipole limit to the limit where the anisotropy energy is dominant. We have obtained the Smoluchowski equation describing the evolution of the probability density of the relevant degrees of freedom of the particles. This equation allows us to obtain a hierarchy of dynamic equations for the different correlation functions that can be closed using appropriate decoupling approximations. The correlation dynamics provides expressions for the characteristic relaxation times and they constitute the starting point to determine the transport coefficients using the GreenKubo formulas. It is interesting to emphasize that the 
theory we have developed encompasses a wide number of situations characterized by the values of the magnetic and anisotropy energies of the ferromagnetic particles.

To check the validity of our formalism, we have compared our results for the relaxation time of the particles to birefringence experiments carried out in two opposite limits: $\sigma \gg \mu$ and $\mu \gg \sigma$. The first case corresponds to a rigid dipole, whereas in the second, the magnetic moment relaxes towards the field very rapidly and independently of the particle axis, which also relaxes towards the magnetic moment in a larger time scale. In both cases, we have obtained quite a good agreement with the experiments.

\section{ACKNOWLEDGMENTS}

We acknowledge A. Pérez-Madrid for fruitful conversations on this subject. This work has been supported by DGICYT (Spain) under Grant No. PB92-0859. One of us (M.C.M.) wishes to thank Generalitat de Catalunya for financial support.

\section{APPENDIX: DECOUPLING APPROXIMATIONS}

In Sec. III, we have introduced decouplings of some correlation functions appearing in the evolution equations for the correlations. The purpose of this appendix is to give more details about the procedure followed to carry out such decouplings. In Eq. (3.4), the approximated quantities are

$$
\begin{aligned}
\left\langle\left(R_{x} R_{z}\right)(t) R_{x}(0)\right\rangle & \sim\left\langle R_{x}(t) R_{x}(0)\right\rangle\left\langle R_{z}\right\rangle_{e q} \\
& =\mathcal{L}(\mu)\left\langle R_{x}(t) R_{x}(0)\right\rangle
\end{aligned}
$$

and

$$
\begin{aligned}
\left\langle\left[R_{x}(\hat{n} \cdot \hat{R})^{2}\right](t) R_{x}(0)\right\rangle & \sim\left\langle R_{x}(t) R_{x}(0)\right\rangle\left\langle(\hat{n} \cdot \hat{R})^{2}\right\rangle_{e q} \\
& =Q(\sigma)\left\langle R_{x}(t) R_{x}(0)\right\rangle,
\end{aligned}
$$

where linearizations in time have been performed. The approximation (A1) was already discussed in the text [see Eq. (3.5) and comments below]. For the correlation
(A2), we decouple the quantities $R_{x}$ and $(\hat{n} \cdot \hat{R})^{2}$ because they are not coupled in equilibrium conditions, $\left\langle R_{x}(\hat{n}\right.$. $\left.\hat{R})^{2}\right\rangle_{e q}=0,\left\langle R_{x}\right\rangle_{e q}=0$, and $\left\langle(\hat{n} \cdot \hat{R})^{2}\right\rangle_{e q} \neq 0$. Thus, in a situation not far from equilibrium, we will assume that both quantities also remain decoupled.

By similar arguments, in Eq. (3.6) we have also performed the following approximations:

$$
\begin{aligned}
\left\langle\left[R_{x}\left(n_{z}(\hat{n} \cdot \hat{R})\right)\right](t) R_{x}(0)\right\rangle & \sim\left\langle R_{x}(t) R_{x}(0)\right\rangle\left\langle\left[n_{z}(\hat{n} \cdot \hat{R})\right]\right\rangle_{e q} \\
& =\mathcal{L}(\mu) Q(\sigma)\left\langle R_{x}(t) R_{x}(0)\right\rangle, \\
\left\langle\left[n_{x}\left(R_{z}(\hat{n} \cdot \hat{R})\right)\right](t) R_{x}(0)\right\rangle & \sim\left\langle\left[n_{x}(\hat{n} \cdot \hat{R})\right](t) R_{x}(0)\right\rangle\left\langle R_{z}\right\rangle_{e q} \\
& =\mathcal{L}(\mu)\left\langle\left[n_{x}(\hat{n} \cdot \hat{R})\right](t) R_{x}(0)\right\rangle,
\end{aligned}
$$

and

$$
\begin{aligned}
& \left\langle\left\{n_{x}(\hat{n} \cdot \hat{R})\left[1-(\hat{n} \cdot \hat{R})^{2}\right]\right\}(t) R_{x}(0)\right\rangle \\
& \quad \sim\left\langle\left[n_{x}(\hat{n} \cdot \hat{R})\right](t) R_{x}(0)\right\rangle\left(1-\frac{\left\langle(\hat{n} \cdot \hat{R})^{4}\right\rangle_{e q}}{\left\langle(\hat{n} \cdot \hat{R})^{2}\right\rangle_{e q}}\right) .
\end{aligned}
$$

In Eq. (3.7), we have used

$$
\begin{aligned}
\left\langle\left(n_{z}^{2} R_{x}\right)(t) R_{x}(0)\right\rangle \sim & \left\langle R_{x}(t) R_{x}(0)\right\rangle\left\langle n_{z}^{2}\right\rangle_{e q} \\
= & \left(\frac{\mathcal{L}(\mu)}{\mu}[1-3 Q(\sigma)]+Q(\sigma)\right) \\
& \times\left\langle R_{x}(t) R_{x}(0)\right\rangle, \\
\left\langle\left(n_{x} n_{z} R_{z}\right)(t) R_{x}(0)\right\rangle \sim & \left\langle\left(n_{x} n_{z}\right)(t) R_{x}(0)\right\rangle\left\langle R_{z}\right\rangle_{e q} \\
= & \mathcal{L}(\mu)\left\langle\left(n_{x} n_{z}\right)(t) R_{x}(0)\right\rangle .
\end{aligned}
$$

Notice that the decoupling in the correlation $\left\langle\left[n_{x}(\hat{n}\right.\right.$. $\left.\left.\hat{R})^{3}\right](t) R_{x}(0)\right\rangle$ of Eq. (A5) is $\left\langle\left[n_{x}(\hat{n} \cdot \hat{R})\right](t) R_{x}(0)\right\rangle \frac{\left\langle(\hat{n} \cdot \hat{R})^{4}\right\rangle_{e q}}{\left\langle(\hat{n} \cdot \hat{R})^{2}\right\rangle_{e q}}$ and not $\left\langle\left[n_{x}(\hat{n} \cdot \hat{R})\right](t) R_{x}(0)\right\rangle\left\langle(\hat{n} \cdot \hat{R})^{2}\right\rangle_{e q}$, which leads to divergencies of the rotational viscosity at small values of $\mu$. As regards this fact, it is worthwhile to emphasize that these approximations are more accurate for moderate and higher values of parameters $\mu$ and $\sigma$. This type of truncation was already proposed by Stratonovich in the context of stochastic processes.
[1] W.T. Coffey, P.J. Cregg, and Yu.P. Kalmykov, in On the Theory of Debye and Neel Relaxation of Single Domain Ferromagnetic Particles, edited by I. Prigogine and S.A. Rice, Advances in Chemical Physics Vol. 83 (Wiley Interscience, New York, 1992), p. 263.

[2] J.T. Waldron, Yu.P. Kalmykov, and W.T. Coffey, Phys. Rev. E 49, 3976 (1994).

[3] Proceedings of the Sixth International Conference on Magnetic Fluids, edited by V. Cabuil, J.-C. Bacri, and R. Perzynski (North-Holland, Amsterdam, 1993).

[4] V.G. Bashtovoy, B.M. Berkovsky, and A.N. Vislovich, Introduction to Thermomechanics of Magnetic Fluids (Springer-Verlag, Berlin, 1988).
[5] T.L. Gilbert, Phys. Rev. 100, 1243 (1955).

[6] M.C. Miguel, J. Bonet Avalos, A. Pérez-Madrid, and J.M. Rubí, Physica A 193, 359 (1993).

[7] W.F. Brown, Phys. Rev. 130, 1667 (1963).

[8] M.A. Martsenyuk, Yu.L. Raikher, and M.I. Shliomis, Zh. Eksp. Teor. Fiz. 65, 834 (1973) [Sov. Phys. JETP 38, $413(1974)]$.

[9] Yu.L. Raikher and M.I. Shliomis, Zh. Prikl. Mekh. Tekh. Fiz. 4, 41 (1974) [J. Appl. Mech. Tech. Phys. 15, 470 (1974)].

[10] M.I. Shliomis and V.I. Stepanov, J. Magn. Magn. Mater. 122, 196 (1993).

[11] J.H. Calderwood and W.T. Coffey, Proc. R. Soc. London, 
Ser. A 356269 (1977).

[12] N.G. van Kampen, Stochastic Processes in Physics and Chemistry (North-Holland, Amsterdam, 1992).

[13] M.I. Shliomis, Zh. Eksp. Teor. Fiz. 61, 2411 (1972) [Sov. Phys. JETP 34, 1291 (1972)].

[14] M. Doi and S.F. Edwards, The Theory of Polymer Dynamics (Clarendon Press, Oxford, 1986).

[15] J.P. McTague, J. Chem. Phys. 51, 133 (1969).

[16] J.-C. Bacri, K. Djerfi, S. Neveu, and R. Perzynski, J.
Magn. Magn. Mater. 123, 67 (1993).

[17] J.-C. Bacri, J. Dumas, D. Gorse, R. Perzynski, and D. Salin, J. Phys. (Paris) Lett. 46, L1199 (1985)

[18] M.C. Miguel, J.M. Rubí, and A. Pérez-Madrid, Physica A 203, 24 (1994).

[19] J.-C. Bacri and R. Perzynski, in Proceedings of the XII Sitges Conference, edited by L. Garrido (Springer-Verlag, Berlin, 1993). 